\title{
MANEJO LAPAROSCÓPICO DE LA ENDOMETRIOSIS DEL SEPTO RECTOVAGINAL
}

\author{
LAPAROSCOPIC MANAGEMENT OF \\ RECTOVAGINAL SEPTUIM ENDOMETRIOSIS \\ Jaime Saavedra, M.D.* \\ Recibido: noviembre 18/2002 - Revisado: noviembre 25/2002 - Aceptado: julio 25/2003
}

\section{RESUMEN}

La experiencia demuestra que la obliteración del fondo de saco de Douglas secundaria a endometriosis puede ser tratada en forma efectiva por laparoscopia, mediante disección del fondo del saco con escisión de la endometriosis profunda fibrótica y restauración de la anatomía del fondo de saco. De ello resulta la resolución de la infertilidad, del dolor pélvico y la hipermenorrea en la mayoría de los casos.

Entre los beneficios para el paciente se incluye el evitar una cirugía abdominal mayor con la morbilidad relacionada, o la terapia de supresión ovárica que evita la fertilidad durante su administración y además parece no actuar en forma efectiva sobre las lesiones de endometriosis infiltrantes.

El manejo laparoscópico puede ser diferido debido a la naturaleza persistente de la enfermedad, que puede dictar más de una aplicación. Por lo anterior, los factores determinantes para lograr

Profesor Titular Universidad del Valle. Cali, Colombia. Jefe del Servicio de Ginecología. Hospital Universitario del Valle. Cali, Colombia. Presidente de la Sociedad Colombiana de Médicos Endoscopistas. Director del Programa de entrenamiento de Cirugía Endoscopica Ginecológica, Clinica Los Andes. Cali, Colombia. Director General del Centro de Biomedicina Reproductiva del Valle S. A., Fecundar S.A. Cali, Colombia. el resultado deseado son una combinación entre la experticia quirúrgica, y la tenacidad y persistencia de la paciente.

En cuanto a las contraindicaciones del procedimiento, la principal es la inexperiencia de quien lo realiza, ya que esta técnica quirúrgica puede ser más exigente que una histerectomía radical o una cirugía de cáncer de ovario. Quienes recomiendan vaporización de la endometriosis del septum rectovaginal deberían evitar esta operación y hacer una adecuada remisión.

Palabras clave: endometriosis, cirugía laparoscópica, septum rectovaginal

\section{SUMMARY}

The experience shows that obliteration of the cul-de-sac secondary to endometriosis can be effectively treated laparoscopically by cul-de-sac dissection with excision of deep fibrotic endometriosis and restoration of cul-de-sac anatomy resulting in resolution of infertility, pelvic pain, and hypermenorrhea in most cases.

Patient benefits include avoidance of major abdominal surgery with its related morbidity or ovarian suppressive therapy that prohibits fertility during administration and doses not appear to penetrate deep, infiltrating endometriotic lesions. 
The laparoscopic approach can be lengthy, and the persistent nature of the disease may dictate more than one application. Therefore, significant determining factors in achieving the desired outcome may be a combination of surgical skill and tenacity and patient persistence.

The major contraindication of this procedure is the inexperience of the surgeon. Surgeon advocating vaporization of endometriosis should avoid this operation and make an appropriate referral. This surgery can be more demanding than radical hysterectomy or ovarian cancer operation.

Key words: endometriosis, laparoscopic surgery, rectovaginal septum

\section{CARACTERÍSTICAS GENERALES}

\section{DE LA ENDOMETRIOSIS}

La creencia general es que la endometriosis es causada por la implantación de células endometriales de la menstruación retrograda o metaplasia. En la pelvis, se pueden considerar tres formas diferentes de endometriosis:

1. Peritoneal ${ }^{1}$

2. Ovárica ${ }^{2,3}$

\section{Septo rectovaginal ${ }^{4-11}$}

Se cree que las lesiones sutiles no coloreadas son las manifestaciones tempranas de la enfermedad, como son las lesiones blancas (manchas opacificadas, parches amarillo-café, parches de hemosiderina). La baja actividad mitótica en las lesiones blancas sugiere que son una manifestación de la enfermedad. ${ }^{4}$ La presencia de escaso estroma y una pobre vascularizacion del estroma son otros dos argumentos que apoyan esta afirmación.

Las lesiones rojas son formas más activas de la enfermedad, y progresan a las lesiones negras las cuales se pueden considerar como implantes encerrados por tejido fibrótico. Los quistes con flui- do achocolatado son, de acuerdo con la hipótesis de Hughesdon ${ }^{2}$ la consecuencia de la invaginación de implantes superficiales dentro del ovario.

Los endometriomas se pueden desarrollar en los ovarios y este tipo de endometriosis quística se considera como una de las formas severas de endometriosis, a menudo relacionada con infertilidad.

La tercera forma de la enfermedad es la adenomiosis del septo rectovaginal, tal como lo definieron Donnez y colaboradores. ${ }^{8-11}$ y Nisolle y colaboradores. ${ }^{6}$

\section{OBLITERACIÓN PARCIAL O COMPLETA DEL FONDO DE SACO DE DOUGLAS}

Sampson ${ }^{12}$ definió la obliteración del fondo de saco de Douglas como extensas adherencias que sellan su parte inferior y unen la cerviz o la parte inferior del útero al recto; con un adenoma de tipo endometrial que invade el tejido cervical y uterino, y probablemente la pared anterior del recto (en menor grado).

La obliteración del fondo de saco de Douglas secundaria a endometriosis implica la presencia de lesiones fibróticas retrocervicales y profundas, por debajo del peritoneo. Esta variedad se encuentra localizada sobre, o invade la parte anterior del recto, la región posterior de la vagina, o bien el segmento posterior del cerviz, el septo rectovaginal y los ligamentos uterosacros.

\section{DIAGNÓSTICO}

La inspección cuidadosa del fondo de saco de Douglas es necesaria para evaluar la extensión hacia arriba de la tienda que forma el recto. Para determinar si el fondo de Douglas está parcial o totalmente obliterado se inserta una pinza con una gasa doblada dentro del fórnix posterior de la vagina, e insertamos también una sonda en el recto (figura 1). 
1. El Fondo de saco de Douglas normal mostrará la protuberancia que forma la gasa en el fórnix posterior de la vagina, y los ligamentos uterosacros son claramente visibles (figura 2).

2. La obliteración parcial ocurre cuando la tienda rectal es visible, pero la protuberancia de la esponja en el fórnix posterior apenas es visible entre el recto y la U invertida de los ligamentos uterosacros (figura 3).

3. La obliteración completa del fondo de saco de Douglas implica que el contorno del fórnix pos-

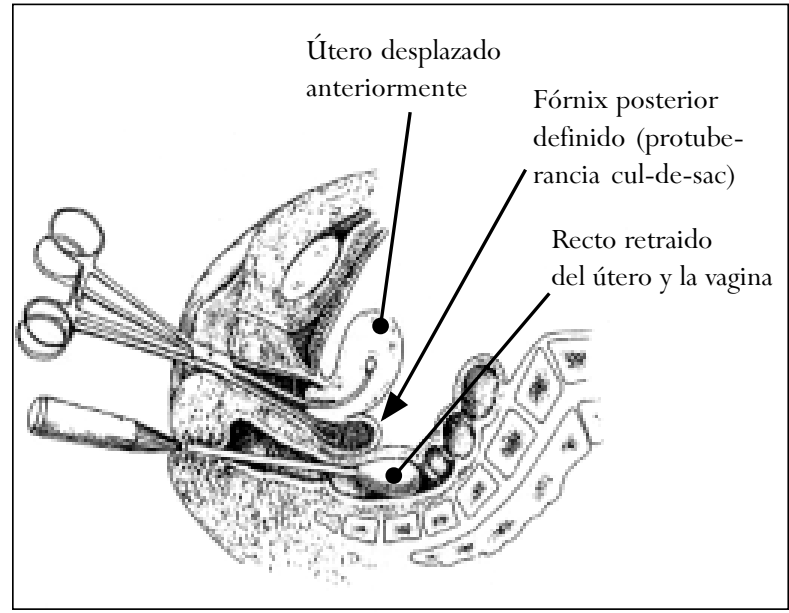

Figura 1. Examen del fondo de saco de Douglas.

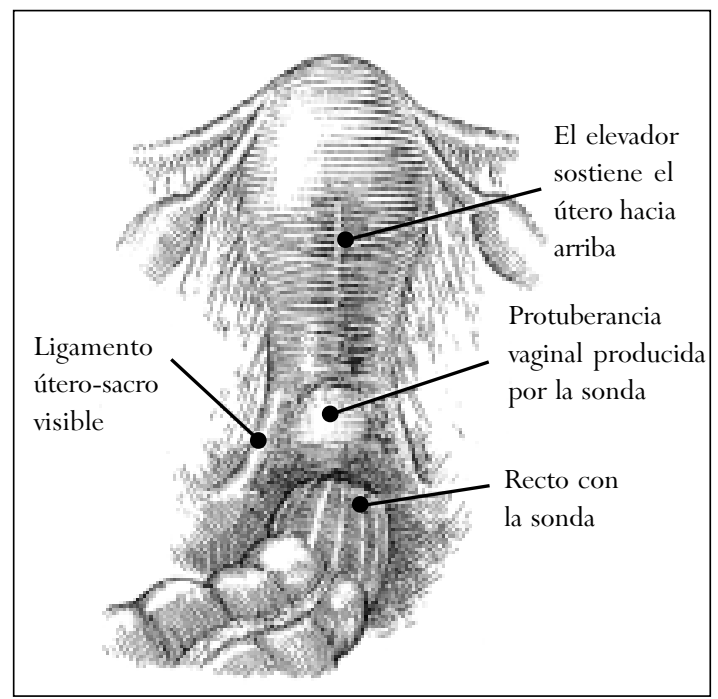

Figura 2. Fondo de saco de Douglas normal. terior no se puede visualizar inicialmente a través del laparoscopio. El recto o los nódulos fibróticos endometriales no permiten identificar la parte profunda del fondo de saco de Douglas (figura 4).

Las opciones de tratamiento para dolor o infertilidad secundaria a la obliteración del fondo de saco de Douglas incluyen terapia de supresión ovárica con danazol o agonistas de la GnRH, o cirugía. ${ }^{13}$

En los casos de infertilidad o cuando se desea preservar la fertilidad, es obligado considerar la cirugía reconstructiva ya sea a través de laparotomía,

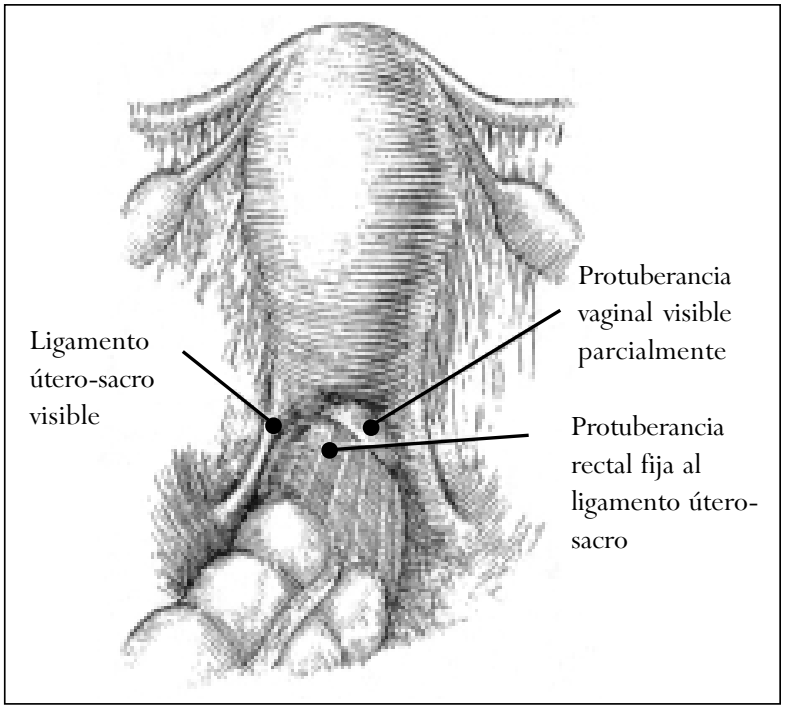

Figura 3. Obliteración parcial del fondo de saco de Douglas.

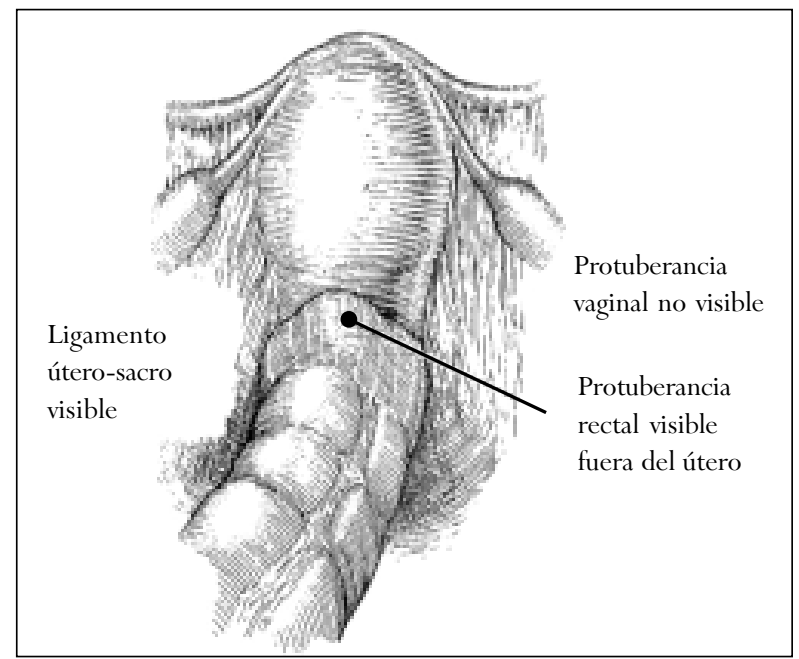

Figura 4. Obliteración completa del fondo de saco de Douglas. 
microcirugía o laparoscopia, dependiendo de la habilidad y experiencia del cirujano.

La endometriosis retrocervical profunda usualmente es manejada por resección intestinal, asumiendo que la mayor parte de la lesión infiltra el recto anterior. En tales casos, la lesión fibrótica profunda es movilizada, comenzando con la parte posterior del útero y progresando hacia abajo donde el recto parece estar pegado. No obstante, se han desarrollado técnicas endoscópicas para tratar esta patología. ${ }^{8-11,14-18}$

\section{RAZONES PARA}

\section{EL MANEJO ENDOSCÓPICO}

\section{Mejor visualización}

El dolor que experimentan estas pacientes es la razón inicial para la cirugía de la endometriosis del fondo de saco de Douglas, siendo mejor la escisión que la ablación superficial de las lesiones infiltrantes profundas, para un efectivo tratamiento de esta condición. ${ }^{15}$

Aunque muchos cirujanos prefieren asumir los riesgos de una laparotomía, nosotros creemos que la experticia en definición anatómica, disección y escisión de las lesiones se pueden realizar eficazmente por laparoscopia; en la cual una mejor visualización compensa la maniobrabilidad quirúrgica restringida.

Los nódulos se pueden palpar con el dedo del cirujano colocado en el recto durante la laparoscopia, y un buen sentido táctil también es posible a través de una sonda laparoscópica. Los limites del manejo laparoscópico de la endometriosis rectal son la pericia y experiencia del cirujano, así como el tiempo requerido para un manejo adecuado.

\section{Ventajas quirúrgicas}

Las ventajas quirúrgicas del manejo laparoscópico de la obliteración del fondo de saco de Dou- glas incluyen acceso intraoperatorio fácil al recto y la vagina, una magnificación que es fácil de manipular con un microscopio operatorio, y la capacidad de llevar a cabo un examen debajo del agua al final del procedimiento, durante el cual todos los coágulos pueden ser evacuados y obtener una completa hemostasia.

Las ventajas generales de la laparoscopia incluyen diagnóstico y tratamiento el mismo día, hospitalización corta, rápida recuperación, excelente aceptación por el paciente, costo efectividad y resultados por lo menos iguales a los de la laparotomía.

En nuestra experiencia, la obliteración del fondo de saco de Douglas secundaria a endometriosis se puede tratar laparoscópicamente por disección del fondo de saco, con escisión de la endometriosis profunda fibrótica, dando como resultado resolución de la infertilidad, del dolor pélvico y de la hipermenorrea en muchos de los casos.

Los beneficios para el paciente son: evitar una cirugía abdominal mayor con su morbilidad relativa, o la terapia de supresión ovárica que impide la fertilidad durante el tiempo de administración y no parece ser útil en el tratamiento de la endometriosis profunda e infiltrante.

\section{Escisión}

La endometriosis extensa de fondo de saco de Douglas se puede controlar laparoscópicamente si el cirujano está dispuesto a gastar tiempo. Si la endometriosis es la única causa del dolor, la remoción laparoscópica a menudo produce mejoría del dolor, a pesar de la severidad de la endometriosis. El danazol rara vez mejora el dolor cuando existen endometriomas ováricos o endometriosis profunda retrocervical. ${ }^{19}$

Experticia quirúrgica y tenacidad son las llaves del éxito en el tratamiento de la endometriosis infiltrante. 


\section{RAZONES EN CONTRA}

\section{Tratamiento inadecuado}

La endometriosis ocasionalmente es una enfermedad que penetra profundamente, es decir, no está limitada superficialmente al tejido. Cuando esto pasa con el recto, el cirujano laparoscopista puede estar tentado a realizar una ablación superficial y considerar la enfermedad virtualmente curada. Esta condición requiere palpación, lo cual siempre es posible en la laparoscopia pero rara vez se realiza, con el fin de apreciar la profundidad del compromiso glandular y la fibrosis en el recto. Por tanto muchos cirujanos pueden tratar la enfermedad superficialmente, dejando porciones profundas de la enfermedad sin tratamiento, lo cual conduce eventualmente a una nueva laparotomía.

\section{Tiempo y experticia}

Se asume que el cirujano laparoscópico es experto en determinar la extensión y profundidad de la enfermedad y es capaz de resecar la lesión, pero la enfermedad puede penetrar la pared del intestino hasta llegar a la mucosa. La reparación del defecto dejado después de la incisión podría requerir sutura. Aunque estas técnicas existen pueden estar muy por encima del promedio de los laparoscopistas en cuanto a la pericia del cirujano y el tiempo quirúrgico deseado. Por eso muchas veces es aconsejable abandonar el enfoque laparoscópico en favor de la técnica de la laparotomía abierta, siendo de esta forma la escisión de la lesión rectal más rápida y segura.

\section{OBJETIVOS DE LA CIRUGÍA}

Para preservar la fertilidad, se deben considerar los procedimientos reconstructivos, ya sea microcirugía por laparotomía o por laparoscopia, dependiendo de la pericia y experiencia del cirujano.

Para dolor, cuando no se desea fertilidad futura, se debe considerar la escisión del tejido endo- metriósico; es fácil resecar este tejido de la vejiga, vagina y recto si el útero no tiene que ser removido. No obstante la histerectomía con salpingooforectomia bilateral se realiza con frecuencia. El problema con este enfoque es que la histerectomía usualmente se realiza con la técnica intrafasial, dejando la endometriosis fibrótica sobre la vagina y el recto, asumiendo que ello se resolvería después de la castración. Con el tiempo será necesario practicar una nueva intervención para mejorar el dolor que produce la endometriosis ubicada en el mango vaginal o el recto.

En mi enfoque, primero se libera el recto anterior disecándolo a través del tejido fibrótico, mientras se identifica el contorno del recto con el uso de una sonda roma colocada en su interior. Esto es posible aún si la parte anterior del recto es infiltrada por la endometriosis. En muchos casos, después de realizar lo anterior, la masa grande de endometriosis fibrótica se visualiza en la parte posterior de la vagina.

En el enfoque por laparotomía, la endometriosis fibrótica retrocervical profunda se ha manejado usualmente por resección intestinal, asumiendo que parte de la lesión infiltra el recto anterior. En estos casos la lesión fibrotica profunda se moviliza comenzando en la parte posterior del útero y avanzando hacia el recto hasta donde éste se encuentra adherido.

La resección intestinal por laparotomía o laparoscopia en algunos de los casos es necesaria. Sin embargo, es prudente evitar y no estimular el uso de procedimientos agresivos y potencialmente mórbidos.

\section{INDICACIONES DE LA DISECCIÓN DEL FONDO DE SACO DE DOUGLAS}

La principal indicación para disecar el fondo de saco de Douglas obliterado parcial o totalmente es el 
dolor pélvico. Con menos frecuencia se incluye la hipermenorrea y la presencia de una masa pélvica asintomática. Los diagnósticos son realizados por medio de una laparoscopia diagnóstica o provienen de reportes quirúrgicos efectuados por otros cirujanos. Infortunadamente, este diagnostico algunas veces es pasado por alto en la laparoscopia diagnóstica. El índice de sospecha para la endometriosis del septo recto vaginal o intestinal debe ser alto cuando el paciente se queja de dispareunia profunda, dolor con los movimientos intestinales, espasmo rectal o sangrado rectal con la menstruación.

\section{Contraindicaciones}

Son pocas las contraindicaciones para la disección del fondo de saco de Douglas, aún en mujeres con historia de cirugías abdominales previas que han sido tratadas laparoscópicamente sin complicaciones.

La mayor contraindicación es la inexperiencia del cirujano. Son pocos los cirujanos que están preparados física y mentalmente para realizar una disección del fondo de saco de Douglas con escisión de endometriosis fibrótica ya sean por laparoscopia o laparotomía. Quienes abogan por la vaporización de la endometriosis deberían evitar esta cirugía y hacer una remisión apropiada del caso. Esta cirugía puede ser más exigente que la histerectomía radical o una operación de cáncer de ovario.

\section{ASPECTOS CLÍNICOS Y LAPAROSCÓPICOS}

\section{Aspectos clínicos y diagnóstico}

Es importante realizar una radiografía del colon para evaluar el compromiso de la superficie rectal. Aunque las características radiográficas constituyen la mejor forma de evaluar el grado de infiltración de la pared anterior, el uso de la ecografía, vaginal y rectal también es útil en el diagnóstico.
El examen con espéculo de la vagina revela una mucosa normal o la presencia de nódulos azulosos en el fórnix posterior. Por palpación, se puede evaluar el diámetro de lesión y a pesar que este procedimiento puede ser doloroso es necesario realizarlo, por cuanto dicha lesión puede ser causa de dispareunia profunda y dismenorrea.

\section{Aspectos laparoscópicos}

Para determinar si existe obliteración del fondo de saco de Douglas introducimos una torunda de gasa montada en una pinza de anillo dentro del fórnix vaginal posterior (figuras 1-4). Sistemáticamente, se introduce también una sonda rectal. La obliteración completa se diagnostica cuando no es posible visualizar el contorno del fórnix posterior a través del laparoscopio .

\section{TÉCNICA QUIRÚRGICA}

Independientemente del grado de compromiso del saco de Douglas por la endometriosis, se debe realizar una preparación mecánica del intestino administrando oralmente Golytely o Colyte la noche previa al procedimiento, para inducir una diarrea vigorosa y transitoria que limpie rápidamente el intestino sin producir un desequilibrio hidroelectrolítico.

Todo el procedimiento laparoscópico se realiza bajo anestesia general. Para la disección utilizamos láser de $\mathrm{CO}_{2}$, electrocirugía con electrodo de espátula y disección con agua.

La endometriosis nodular profunda que compromete la parte posterior de la vagina, recto, cerviz posterior y ligamentos uterosacros, requiere de una escisión en la forma más completa posible. Como fue descrito por Reich y colaboradores ${ }^{14} \mathrm{y}$ Donnez y colaboradores, ${ }^{8-11}$ la atención se focaliza en la disección del recto anterior a través de su área de compromiso hasta llegar al tejido laxo del espacio rectovaginal. Previamente se ha colocado en el fórnix vaginal posterior una torunda de gasa fija a 
una pinza de anillo, y simultáneamente colocamos una sonda especial en el recto. Adicionalmente, insertamos en la cavidad endometrial una cánula para antevertir el útero. El peritoneo que cubre el fondo de saco de Douglas es abierto entre la lesión adenomiótica y el recto.

Nosotros usamos la técnica de primero liberar el recto anterior del tejido laxo areolar del septo rectovaginal, antes de escindir y/o vaporizar las lesiones visibles, palpables y profundas de la endometriosis.

Realizamos disección cuidadosa utilizando el disector con agua a presión, y láser de $\mathrm{CO}_{2}$ para disección fina, hasta que el recto es completamente liberado e identificable por debajo de la lesión.

La escisión del tejido fibrótico únicamente la realizamos después de que la disección del recto ha sido completa. En caso de lesiones profundas, la pared vaginal puede encontrarse infiltrada por la endometriosis y es necesario escindir parte de la vagina en bloque dejando medio centímetro de bordes libres de lesión. El neumoperitoneo se puede mantener con una sonda foley No. 30 y la pared vaginal posterior se cierra vaginalmente.

El recto anterior se reperitoniza aplicando los ligamentos uterosacros y el peritoneo rectal lateral a través de la línea media usando una sutura 3-0 ó 4-0 de aplicación extracorpórea. ${ }^{14}$

Después del procedimiento de cierre, la hemostasia es verificada relizando una examen bajo agua para detectar sangrado provenientes de vasos taponados durante el procedimiento por la presión intraperitoneal que hace el neumoperitoneo de $\mathrm{CO}_{2}$.

En un estudio realizado por Donnez y colaboradores, ${ }^{18}$ se realizó escisión de nódulos de endometriosis localizados en el fondo de saco de Douglas a 437 pacientes, seguidos por más de 3 años (3 a 10 años). Los pacientes fueron clasificados en 3 grupos:
Grupo 1: Escisión del nódulo sin reseción de la mucosa vaginal (laparoscopia) en 157 pacientes (34\%).

Grupo 2: Escisión del nódulo con reseción del fórnix vaginal posterior (laparoscopia) en 272 pacientes $(62,5 \%)$.

Grupo 3: Escisión del nódulo y reseción intestinal por estenosis subtotal del intestino (laparotomia) en 15 pacientes (3,5\%).

Ellos informaron, de acuerdo con la técnica quirúrgica utilizada en los tres grupos de pacientes, una frecuencia de recurrencia de la dismenorrea de 29\% en el Grupo 1, 17\% en el Grupo 2 y 26\% en el Grupo 3.

La misma conclusión fue presentada para la recurrencia de dispareunia severa.

Para estos autores los mejores resultados se encontraron para el grupo al cual le realizaron escisión del nódulo con resección del fornix posterior de la vagina, incluyendo la resección de los nervios simpáticos situados entre la cerviz y los ligamentos uterosacros. Tales resultados son mejores con respecto a la resección intestinal. Lo anterior comprueba de nuevo que procedimientos muy agresivos, incluyendo la resección intestinal, no necesariamente previenen la recurrencia.

Por consiguiente, la resección del fórnix vaginal se debe considerar como la primera elección, y la resección intestinal está indicada para casos de sangrado rectal o en casos de estenosis del sigmoide o el recto.

\section{DISCUSIÓN}

En algunas lesiones, el principal hallazgo es un intestino adherido sobre la lesión, el cual puede estar localizado profundamente en el septum recto vaginal, aunque no infiltrándolo realmente. Otro tipo de lesión es aquella mas profunda, de forma esférica, situada en septum rectovaginal, y a menudo es visible en la laparoscopia como una pe- 
queña lesión típica de endometriosis. Esta lesión a menudo es más palpable que visible.

Se han descrito dos tipos diferentes endometriosis profunda:

1. La endometriosis verdadera infiltrante que se caracteriza por lesiones peritoneales profundas muy activas en el espacio retroperitoneal. En los casos de invasión peritoneal lateral, se comprometen los ligamentos útero-sacros.

2. Adenomiosis del septum recto vaginal, la cual se origina del apartir del tejido del septum recto vaginal, conformada esencialmente por músculo liso con epitelio glandular activo y escaso estroma. $^{20}$

Kelly y Diamond ${ }^{21}$ reportaron en una serie de 68 mujeres sometidas a laparotomía por endometriosis, que fue necesario penetrar a la luz intestinal para escindir la endometriosis. La endometriosis del colon fue vaporizada hasta llegar al tejido normal, lo que se demostró por palpación y visión con magnificación.

Un procedimiento laparoscópico amplio, aunque no erradique toda la endometriosis, puede resultar en una mejoría notable del dolor o en un embarazo deseado. Algunas veces se acepta que la reseción intestinal puede ser necesaria en casos raros $(1,8 \%)$, pero es prudente evitar y no estimular el uso de procedimientos agresivos, que pueden conducir a una alta morbilidad.

Según Donnez y colaboradores, ${ }^{18}$ la adenomiosis profunda se debe considerar como una enfermedad específica, diferente de la endometriosis mínima o leve y del endometrioma ovárico. Ellos sugieren que esta enfermedad debe ser llamada "adenomiosis recto vaginal".

\section{BIBLIOGRAFÍA}

1. Nisolle M, Casanas - Roux, Anaf V, et al. Morphometric study of the stromal vascularization in peritoneal endometriosis. Fertil Steril 1993; 59:681-684
2. Hughesdon PE. The structure of endometrial cysts of the ovary. J Obstet Gynaecol Br Empire. 1957; 64: $481-487$

3. Donnez J, Nisolle M, Gillet N, et al. Large ovarian endometriomas. Hum Reprod. 1996; 11:641-646

4. Donnez J, Nisolle M, Casanas - Roux F. Three dimensional architectures of peritoneal endometriosis. Fertil Steril. 1992; 57:980-983

5. Nisolle M, Donnez J. Peritoneal endometriosis, ovarian endometriosis, and adenomyotica nodules of the rectovaginal septum are three different entities. Fertil Steril. 1997; 68:585-596

6. Donnez J, Nisolle M. Appearances of peritoneal endometriosis. In Proceedings of The $3^{\text {rd }}$ International Laser Surgery Symposium, Brussels, 1988.

7. Nisolle M, Paindaviene B, Bourdon A, et al. Histologic study of peritoneal endometriosis in infertile women. Fertil Steril 1990; 53:984-988

8. Donnez J, Nisolle M, Casanas-Roux F, et al. Laparoscopic treatment of rectovaginal septum endometriosis. In Donnez J, Nisolle M, Eds. An Atlas of Laser Operative Laparoscopy and Histeroscopy. Cornforth, UK: Parthenon Publishing, 1994: 75-85.

9. Donnez J, Nisolle M. Advanced laparoscopy surgery for the removal of rectovaginal septum endometriotic and adenomyotic nodules. Baillieres Clin Obstet Gynecol 1995,9:769-774

10. Donnez J, Nisolle M, Casanas - Roux F, et al. Rectovaginal septum endometriosis or adenomyosis : laparoscopic management in a series of 231 patients. Human Reprod 1995, 10:630-635

11.Donnez J, Nisolle M, Guillenot S, et al. Rectovaginal septum adenomyotic nodules: a series of 500 cases. Br J Obstet Gynecol 1991; 104:1009-1013

12. Sampson JA. Intestinal adenomas of endometrial type. Arch Surg 1922; 5:217-221

13. Donnez J, Nisolle, Casanas - Roux F. Endometrisosis associated infertility: evaluation of preoperative use of Danazol, gestrinone and buserelin. Int J Fertil $1990 ; 35: 297-301$

14. Reich H, McGlinn F, Salvat J. Laparosocopic tretament of cul-de-sac obliteración secondary to retrocervical deep fibrotic endometriosis J Reprod Med 1991;36:516-521 
15.Knick PD. Deeply infiltrating endometriosis. In Bronsens I, Donnez J, eds. Endometriosis : Research and Management. Carnforth, UK: Parthenon Publishing, 1993 pp:437-446

16. Nezhat C, Nezhat F, Pennington E. Laparoscopic treatment of lower colorectal and infiltrative rectovaginal septum endometriosis by the technique of video laparoscopy. Br J Obstet Gynaecol 1992; 99:664-667

17. Canis M, Wattiez A, Pouly JL, et al: Laparoscopic treatment of endometriosis. In Bronsens I, Donnez J, Eds. Endometriosis: Research and Management. Carnforth, UK: Parthenon Publishing, 1993; pp: 407-417.
18. Donnez M, Nisolle J, et al. Laparoscopic treatment of rectovaginal septum adenomyosis. In An Atlas of Operative Laparoscopic and Histeroscopy. 2nd ed. J: Donnez and M. Nisolle Ed. The Parthenon Publishing Group. London, 2001;pp: 83-92.

19. Telimaa S: Danazol and medroxiprogesterone acetate inefficacious in the treatment of infertility in endometriosis. Fertil Steril. 1988; 50:872-875.

20.Zoloudek C, Norris HJ. Mesenchymal tumors of the uterus. In Kurman R, ed. Blasteins Pathology of the Female Genital Tract. New York, 1987; pp: 373408.

21. Kelly R, Diamond MP. Laparotomy in infertile patients (use of $\mathrm{CO}_{2}$ laser). J Repro Med 1989; 34:25-29. 